\title{
A novel TEX11 mutation induces azoospermia: a case report of infertile brothers and literature review
}

\author{
Yanwei Sha ${ }^{1 \dagger}$, Liangkai Zheng ${ }^{1 \dagger}$, Zhiyong Ji ${ }^{1}$, Libin Mei ${ }^{1}$, Lu Ding ${ }^{1}$, Shaobin Lin', Xu Wang ${ }^{1}$, Xiaoyu Yang ${ }^{2 *}$ \\ and Ping $\mathrm{Li}^{1 *}$ (D)
}

\begin{abstract}
Background: Testis-expressed gene 11 (TEX11) is an X-linked gene and essential for meiotic recombination and chromosomal synapsis. TEX11 deficiency causes meiotic arrest and male infertility, and many TEX11 mutations have been found in azoospermic and infertile men.
\end{abstract}

Case presentation: This study reported one novel TEX11 mutation (2653G $\rightarrow T$, in exon 29, GenBank accession number, NM_031276) in two brothers with azoospermia. This mutation was firstly screened out by whole-exome sequencing (WES) and further verified by amplifying and sequencing the specific exon 29. Surprisingly, the same exonic missense mutation (W856C) was observed in two brothers but not in their mother. Histological analysis of testicular biopsy from both brothers revealed meiotic arrest and no post-meiotic round spermatids and mature spermatozoa were observed in the seminiferous tubules. TEX11 expression was observed strongly in spermatogonia and weakly in spermatocytes, but not in Sertoli cells and interstitial cells.

Conclusions: We identified one novel TEX11 mutation in two brothers and summarized the literature regarding TEX11 mutations and male infertility. This study and previous literature indicate that TEX11 mutations are closely associated with male infertility, especially azoospermia, although auxiliary clinical analyses are needed to figure out the causes of male infertility.

Keywords: TEX11 mutation, Azoospermia, Infertility, Meiosis, Whole-exome sequencing

\section{Background}

Infertility is defined as the inability to conceive after 12 months or more of unprotected sexual activity and is a major reproductive health problem $[1,2]$. Infertility affects $10-15 \%$ of couples, and about $20-30 \%$ of infertility is caused by male factors. In humans, male infertility such as azoospermia or severe oligospermia is mostly characterized by decreased semen parameters [3]. The majority $(\sim 75 \%)$ of male patients with spermatogenic failure are idiopathic, and a genetic factor is often considered as the major causes $[4,5]$. The most common strategies for the genetic diagnosis of male

\footnotetext{
*Correspondence: yxy1921@163.com; saarc2001@sina.com

${ }^{\dagger}$ Equal contributors

${ }^{2}$ Department of Reproductive Medicine, First Affiliated Hospital of Nanjing Medical University, Nanjing 210029, Jiangsu Province, China

'Reproductive Medicine Center, Xiamen Women's and Children's Health

Hospital, Xiamen 361003, Fujian Province, China
}

infertility are to screen for the presence of chromosomal aberrations, long arm of the $\mathrm{Y}$ chromosome (Yq) microdeletions, and gene mutations. In all cases of male infertility, karyotype abnormalities and Yq microdeletions are detected in $\sim 5 \%$ and $\sim 7.4 \%$ of patients, respectively [6]. In men with azoospermia, the prevalence of these two aberrations obviously increases to $>13 \%$ (chromosomal aberrations) and $>10 \%$ (Yq microdeletions) [6]. Yq microdeletions mainly occur in three or four "azoospermia factor" regions: AZFa, AZFb, AZFc, and AZFd (potential) [7]. It has been demonstrated that germ cellspecific genes are enriched in the mammalian $\mathrm{X}$ chromosome [5]. Many X-linked genes are expressed in the testis, and their functions in spermatogenesis have gradually been recognized in knock-out models and subjects with mutations. Three X-linked genes including testis-expressed gene 11 (Tex11), TATA-box binding 
protein-associated factor 7 like (Taf7l) gene, and nuclear RNA export factor $2(N x f 2)$ are known as important regulators of male fertility in mouse models [8-10]. The Tex11 gene is conserved in vertebrates and encodes a $104-\mathrm{kDa}$ protein with a tetratricopeptide repeat motif that mediates protein-protein interactions [11, 12]. TEX11 can form distinct foci on meiotic chromosomes in spermatocytes and oocytes, thus it is considered as a meiosis-specific factor [13]. TEX11 transcripts are exclusively expressed in the testis, and TEX11 protein is observed in the cytoplasm and nuclei of type B spermatogonia, with the highest level in zygotene spermatocytes, and a basal level in late pachytene spermatocytes $[14,15]$. The abundant expression of TEX11 protein in type B spermatogonia and early spermatocytes supports that TEX11 plays a key role in the early stage of germ cell development. In addition, Yang et al. have reported that male TEX11 $1^{-1-}$ mice are infertile due to meiotic arrest [13]. Both Nxf2- and Taf7l-knockout mice exhibit reduced semen parameters and impaired motility $[9,10]$. A kinase anchor protein 4 (Akap4)-deficient mice are infertile due to a poorly developed fibrous sheath and a short flagellum in the spermatozoa [16]. The androgen receptor gene on the long arm of the $\mathrm{X}$ chromosome has been found to play a role in meiosis and the conversion of spermatocytes to round spermatids during spermatogenesis [17]; while its mutation leads to androgen insensitivity syndrome and Kennedy syndrome, a neurodegenerative disorder with spermatogenesis abnormalities [18]. Moreover, mutations or partial deletions of some $\mathrm{X}$-linked genes such as $A K A P 3$, AKAP4, NXF2, TAF7L, USP26, and TEX11 are linked to male subfertility or infertility [19-21].

In this literature review, we mainly discuss the dominant effects of TEX11 on spermatogenesis and male infertility. Specific expression of TEX11 protein in spermatogonia and spermatocytes suggests that TEX11 may play a role in the early stage of germ cell development $[14,15]$. Interestingly, a recent study by $\mathrm{Yu}$ et al. has reported a negative effect of TEX11 on the proliferation of germ-cell-derived GC-1 and GC-2 cells [22]. They have found that TEX11 suppresses the phosphorylation of AKT and ERK signaling pathways through inhibiting ER $\beta$ binding to hematopoietic pre-B cell leukemia transcription factor-interacting protein [22]. Moreover, Tex11 has been identified as the first X-encoded meiosis-specific factor in mice. In another study, Tang et al. have reported that Tex11 is also exclusively expressed in germ cells in the adult pig testis. The expression of porcine Tex11 is correlated with the onset of meiosis, and the expression pattern of TEX11 homologs is highly conserved between pig and mouse [23]. Additionally, single nucleotide polymorphisms (SNPs) have a major impact in percentage of normal sperm, SNPs in TEX11 gene showed close association with idiopathic male infertility [24-26]. After the generation of Tex11-deficient mice, the functions of the Tex11 gene started to be uncovered. In 2008, Yang et al. generated Tex11-null mice by deleting 27 of the total 30 exons in the Tex11 gene [13]. In TEX11-deficient mice, spermatogenesis is impaired due to chromosomal asynapsis at the pachytene stage and reduced crossover formation at the anaphase I stage [13]. TEX11-deficient spermatocytes mostly undergo apoptosis at the pachytene stage, while survived cells display chromosome nondisjunction at the first meiotic division, which causes cell death and male infertility [13]. Interestingly, another group generated a Tex11 mutant strain by deleting exon 3, and found that the mutant males and females showed normal fertility, but the mutant males exhibited delayed repair of double-strand breaks (DSBs) in spermatocytes [14]. DSB repair and chromosome synapsis exert key roles in maintaining genetic integrity, while their malfunctions will cause various diseases such as infertility. In TEX11 mutant (exon 3 deletion) mice, spermatocytes exhibited delayed repair of DSBs and decreased crossover formation [14]. Due to the unique hemizygous and transcriptional status of the $\mathrm{X}$ chromosome, it is wellrecognized that the mutations in single-copy $\mathrm{X}$-linked genes cannot be compensated by a corresponding wild-type allele [4]. Recently, X-linked TEX11 mutations have been observed in infertile men [4, 21]. Yang et al. sequenced the amplicons covering the TEX11 exons 2-30 and flanking intronic regions in a large cohort of infertile men with nonobstructive azoospermia as well as fertile controls. They observed a total of 40 different sequence variants in the introns and exons of TEX11 [4]. Among them, 21 variants were singletons (only observed in one man, 18 variants in infertile men), while 19 variants were observed in 2 or more infertile men and/or fertile controls [5]. Moreover, another recent study screened for mutations in the TEX11 open reading frame in 289 patients with azoospermia and 384 controls [21]. They identified the loss of three TEX11 exons (exons 10-12) in two patients with azoospermia and five novel TEX11 mutations (three splicing mutations and two missense mutations) in 7 of 289 (2.4\%) men with azoospermia [21]. Therefore, the identification of mutations in X-linked genes essential for fertility may be important to figure out the underlying causes of male infertility, especially in men with azoospermia or severe oligospermia.

In the present study, we reported a case of two sterile brothers due to severe nonobstructive azoospermia and analysed the genetic causes by whole-exome sequencing 
(WES). In addition, we summarized the literature regarding TEX11 mutations and male infertility.

\section{Case presentation}

\section{Patients and medical examinations}

This study was approved by the Ethics Committee of Xiamen Women's and Children's Health Hospital. Written consent was obtained from two brothers and their mother, and the family member of two fertile controls who died from car accident. Two infertile brothers attended the Reproductive Medicine Center of Xiamen Women's and Children's Health Hospital (Fujian Province, China) due to a history of primary infertility that had lasted for longer than 2 years. A questionnaire as well as standardized physical, clinical, and laboratory examinations were carried out to record details of their lifestyle, habits, occupation, marriage, family history, physical information (height and body weight), and chromosome and hormone levels (listed in Table 1). There was no history of genetic diseases or infertility in their family. Their parents had a nonconsanguineous marriage. The medical history of both spouses was unremarkable, and all parameters of the female medical check-up were normal. To verify their azoospermia status, three semen analyses (1 week interval) were carried out after 3 days of sexual abstinence, according to WHO guidelines [27]. No sperm was found in each round of analyses, so the two brothers were diagnosed as having azoospermia.

\section{Cytogenetic and molecular genetic analyses}

To screen the chromosomal status, cytogenetic chromosomal karyotype analysis and a fluorescent in situ hybridization (FISH) assay were conducted in two brothers and their mother using the peripheral blood cells. Karyotype and FISH analyses were carried out as described previously [28]. The VYSIS AneuVysion DNA Probe Kit (Abbott Laboratories. Abbott Park, IL, USA) was used (incorporating CEP probes for chromosomes 13 and 21 and LSI probes for chromosome X, Y and chromosome 18). A total of 20-100 metaphase cells were analyzed by the G-banding method according to ISCN 2013 guidelines [29], and the chromosome length consisted of approximately 450-550 sub-bands. FISH analysis was conducted with the combination of $S R Y$ and DYZ3 probes. Moreover, screening of Y chromosome deletions in two brothers were analyzed by a real-time PCR method as described previously [19]. According to the European Academy of Andrology and the European Molecular Genetics Quality Network guidelines, three selected sequence-tagged sites within specific AZFa, $\mathrm{AZFb}$, and AZFc regions were chosen as targets. The $S R Y$ region was also examined. Four hydrolysis probes were designed to detect the four amplicons, respectively. The assay was carried out in a quadruplex reaction.

According to the results of the karyotype and FISH analyses, the karyotype of both brothers was normal (46, $\mathrm{XY}$ ), and no gonadal mosaicism was observed although we observed gonadal mosaicism in the mother of both brothers. Moreover, we did not observe any Y chromosome microdeletions in either brothers.

To determine possible mutations causing azoospermia, whole-exome sequencing (WES) in two brothers and their mother was conducted as described previously [30]. Briefly, genomic DNA from two brothers' semen and their mother's blood cells was prepared in Illumina paired-end libraries and sequenced by using the Illumina HiSeq 2000

Table 1 The clinicopathological variables of two infertile brothers

\begin{tabular}{|c|c|c|}
\hline & $\begin{array}{l}\text { Patient } 1 \\
\text { Value (normal range) }\end{array}$ & $\begin{array}{l}\text { Patient } 2 \\
\text { Value (normal range) }\end{array}$ \\
\hline$\overline{\text { Age }}$ & 30 years old & 29 years old \\
\hline Time of marriage & April 2012 & August 2013 \\
\hline Height & $166 \mathrm{~cm}$ & $169 \mathrm{~cm}$ \\
\hline Body weight & $61 \mathrm{~kg}$ & $66 \mathrm{~kg}$ \\
\hline occupation & soldier & salesman \\
\hline Testicular volume & $\sim 15 \mathrm{~mL}$ & $\sim 15 \mathrm{~mL}$ \\
\hline Lateral spermatic vein & normal & normal \\
\hline Chromosome & $46, X Y$ & $46, X Y$ \\
\hline Y chromosome microdeletion & Not detected & Not detected \\
\hline Follicle-stimulating hormone & $5.92 \mathrm{mlU} / \mathrm{mL}(1.27-19.26)$ & $6.25 \mathrm{mlU} / \mathrm{mL}$ (1.27-19.26) \\
\hline Luteinizing hormone & 4.33 mIU/mL (1.24-8.62) & 5.27 mlU/mL (1.24-8.62) \\
\hline Testosterone & $3.06 \mathrm{ng} / \mathrm{mL}(1.75-7.81)$ & $6.57 \mathrm{ng} / \mathrm{mL}(1.75-7.81)$ \\
\hline Estradiol & 22 pg/mL (10-60) & $51 \mathrm{pg} / \mathrm{mL}(10-60)$ \\
\hline Prolactin & 23.43 ng/mL (2-18) & 9.93 ng/mL (2-18) \\
\hline
\end{tabular}


platform. The data were processed and analyzed, according to previous protocols [30]. To screen specific mutations, all variants of the genomewide data were compared to external publicly available databases including the 1000 Genomes Project (http://www.1000genomes.org) and other large-scale exome sequencing projects. Since TEX11 is an X-linked gene, we did not examine the TEX11 allele in the father of the two brothers.

A mutation $(2653 \mathrm{G} \rightarrow \mathrm{T}$, GenBank accession number, NM_031276) in the exon 29 of the TEX11 gene in the X chromosome was identified by WES in two brothers. However, this mutation was not observed in their mother's WES. To verify the mutation identified by WES, exon 29 of the TEX11 gene was amplified from genomic DNA of two brothers and their mother using conventional end-point polymerase chain reaction (PCR) and the following primers: forward, 5'-CTTGCTATGG AACATTCTACAG-3'; reverse, 5'-TGAAGGAGGTA AGGTGGTTA-3'. The PCR product with the appropriate size was observed in both brothers and their mother (Fig. 1a). The sequences of the PCR products were verified by Sanger sequencing. Consistent with the results of WES, the mutation $(2653 \mathrm{G} \rightarrow \mathrm{T})$ was verified in two brothers but not in their mother (Fig. 1b). Accordingly, the change of amino acids was determined to be W856C.

\section{Histological analysis}

To determine the relationship between the histological change and azoospermia, testicular biopsies were performed in two brothers after their approval.
Testicular tissues were fixed in $4 \%$ formaldehyde at $4{ }^{\circ} \mathrm{C}$ for $4 \mathrm{~h}$, dehydrated in graded ethanol, embedded in paraffin, and cut into $4-\mu \mathrm{m}$-thick sections. To examine the testicular histology, the sections were deparaffinized, rehydrated in graded ethanol, and stained by hematoxylin and eosin (H\&E). As a control of normal testicular histology, testicular sections from two fertile men who died from car accident and their body was donated by their family member to our hospital, were obtained from the Pathology Department of our hospital. The seminiferous tubules of fertile testes contained Sertoli cells and a full spectrum of spermatogenic cells including round spermatids and mature spermatozoa. To investigate the effect of TEX11 mutation on the protein expression, we examined TEX11 protein expression in the testicular biopsies from two brothers and a normal testis by immunohistochemistry. Immunostaining of TEX11 was carried out using polyclonal goat-anti-human TEX11 antibody (ab99461, 1:100 dilution, Abcam, Cambridge, MA, USA) on a BenchMark XT automated immunohistochemistry/ FISH slide staining system (Roche Diagnostics (Shanghai) Limited, Shanghai, China), according to the manufacturer's instructions.

Compared with normal testicular histology, the testicular histology from two brothers showed a thicker basement membrane of seminiferous tubules and poorly developed spermatocytes (Fig. 2a). No post-meiotic round spermatids or mature spermatozoa were observed in the seminiferous tubules (Fig. 2a), which is consistent with the typical characteristics of azoospermia. In the
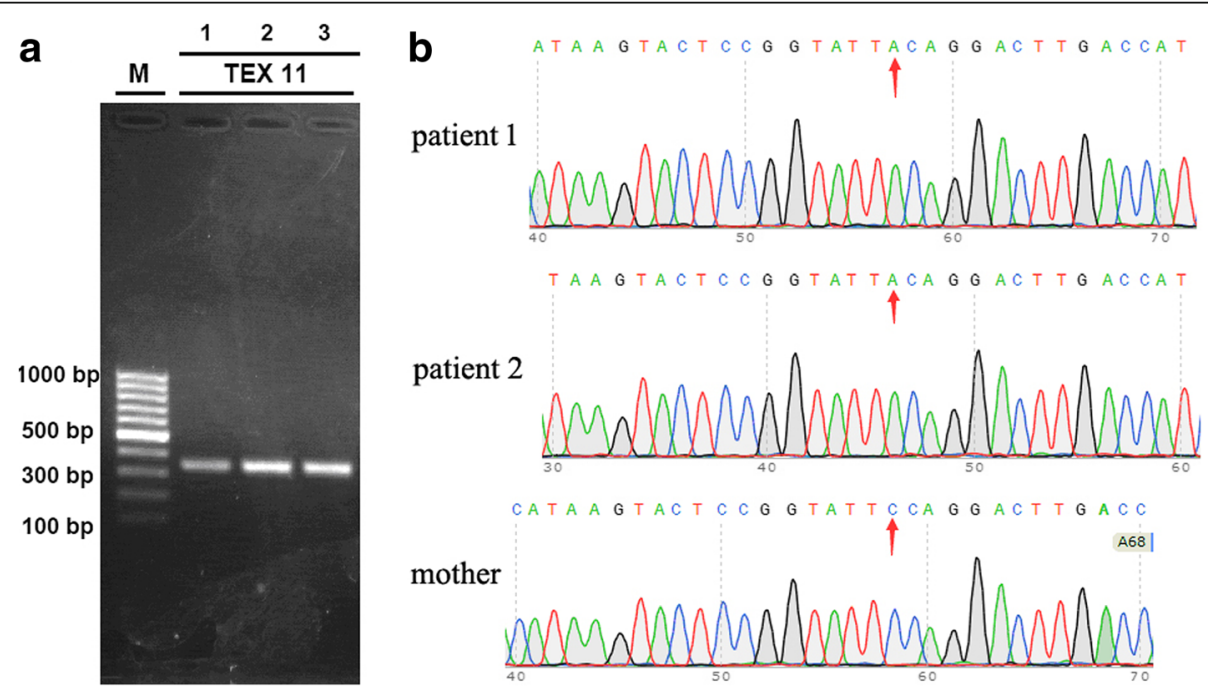

patient 2
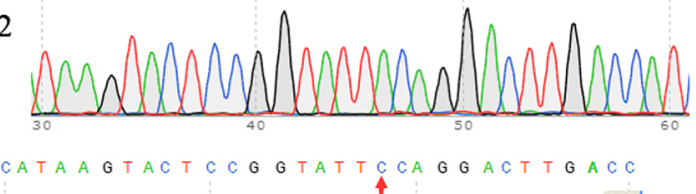

mother
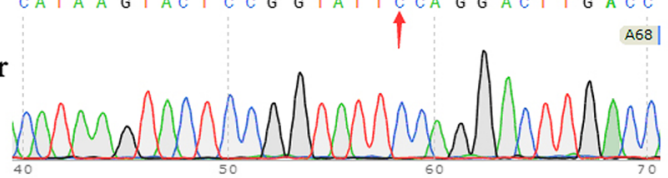

Fig. 1 Identification of the TEX11 mutation. a Amplification of TEX11 exon 29 by PCR. Conventional end-point PCR was performed to amplify exon 29 of the TEX11 gene from genomic DNA of two brothers (lanes 1 and 2) and their mother (lane 3). One clear and specific band at 100 bp was observed. b Mapping of the TEX11 mutation. The PCR product sequences from the two brothers and their mother were verified by Sanger sequencing and aligned to human TEX11 CDNA. Because Sanger sequencing was carried out using the reverse primer, the representative sequences were complementary to human TEX11 cDNA (GenBank accession number, NM_031276). Accordingly, the mutation (C $\rightarrow$ A) in the map was indeed $G \rightarrow T$ in human TEX11 cDNA 


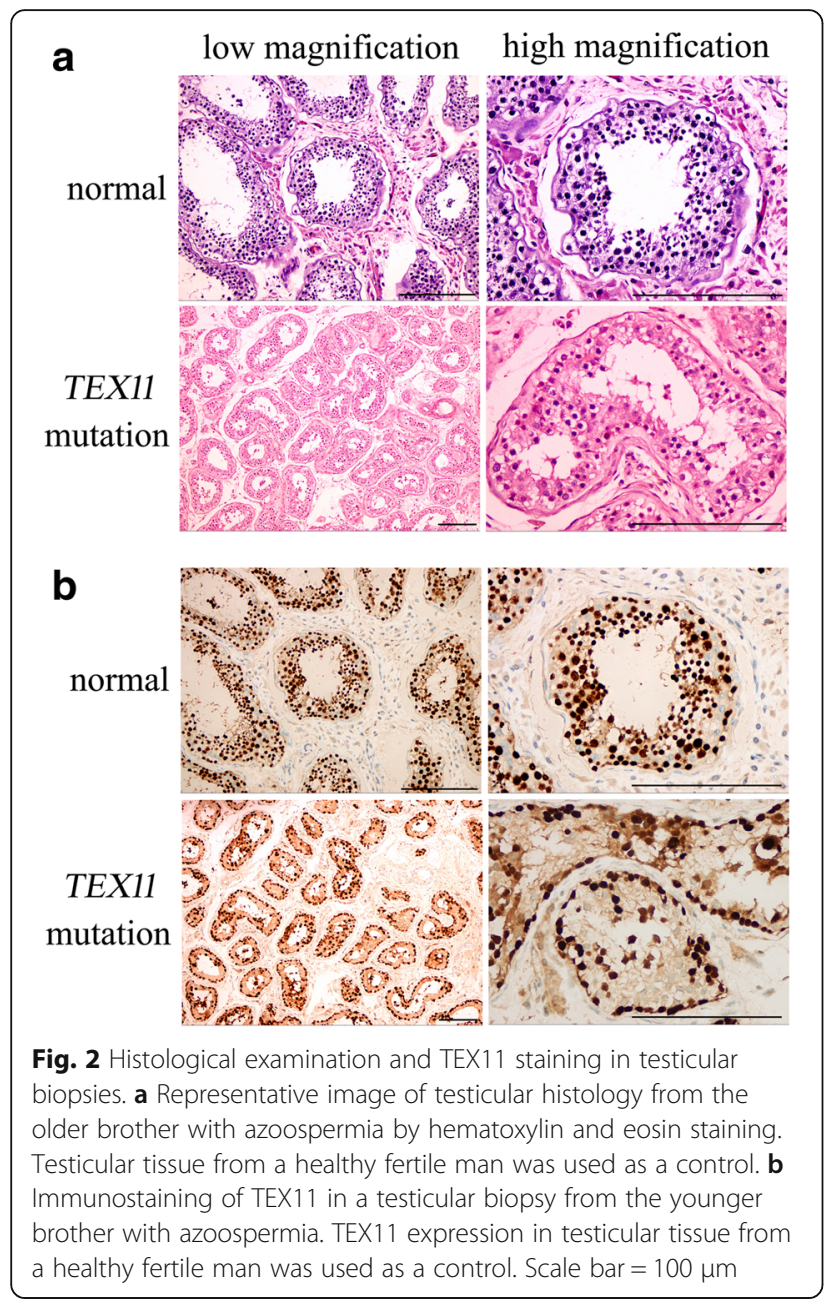

normal testis, TEX11 protein was present in spermatogonia, spermatocytes, round spermatids, and mature spermatozoa in the seminiferous tubules, but it was absent in the surrounding somatic cells including the Sertoli cells and interstitial cells (Fig. 2b). However, in the testicular biopsies from the TEX11-mutated patients, TEX11 protein was observed strongly in spermatogonia and weakly in spermatocytes, while no TEX11 staining was found in Sertoli cells or interstitial cells (Fig. 2b).

\section{Discussion and conclusions}

About $20-30 \%$ of infertility is caused by male factors [31]. The sex chromosomes ( $\mathrm{X}$ and $\mathrm{Y}$ ) in men play key roles in germ cell development. Both chromosomes contain a single copy of genes that are uniquely expressed in male spermatogenesis [20]. In the last decade, extensive efforts have been made to clarify the exact nature of male infertility; however, a large number of infertile men are diagnosed as having "idiopathic infertility". It has been demonstrated that the common genetic causes for male azoospermia are Y chromosome deletion and chromosomal abnormalities such as Klinefelter syndrome (47, XXY karyotype), and these genetic causes are responsible for $\sim 25 \%$ of spermatogenic failure in males $[32,33]$. In the past decade, new technologies including array comparative genomic hybridization (ACGH), single nucleotide polymorphism (SNP) arrays, and nextgeneration sequencing have been used to detect infertility-related genetic defects $[17,20,30]$. A systematic genomic screening of mouse spermatogonia has demonstrated that the genes expressed specifically in male germ cells and located in the $\mathrm{X}$ chromosome play a critical role in early spermatogenesis [15].

Since TEX11 is essential for meiotic recombination and chromosomal synapsis and TEX11 deficiency causes meiotic arrest and male infertility, the identification of TEX11 mutations has become attractive to determine the underlying causes of male infertility, especially in men with azoospermia. It has been found that TEX11 mutations are diverse and present in various forms, such as missense and silent mutations, intronic alterations, frameshift mutations, and hemizygous deletions. Currently, 46 different TEX11 mutations have been identified, including 24 in azoospermic men and 22 in fertile subjects $[4,21]$. The incidence of the TEX11 mutation in azoospermic men from Germany with a European descent was only $2.4 \%$ (7/289), while its prevalence was 14 . $5 \%(35 / 246)$ in American azoospermic males [4, 21]. It would be interesting to know whether this discrepancy is caused by the ethnicity. Two recent studies extensively investigated TEX11 mutations in a large cohort of infertile/azoospermic men [4, 21]. Yatsenko et al. have identified six different TEX11 mutations, including loss of exons 9-11 (607del237bp), three splicing mutations $(405 \mathrm{C} \rightarrow \mathrm{T}, 748+1 \mathrm{G} \rightarrow \mathrm{A}$, and $1793+1 \mathrm{G} \rightarrow \mathrm{C})$, and two missense mutations $(466 \mathrm{~A} \rightarrow \mathrm{G}$ and $2047 \mathrm{G} \rightarrow \mathrm{A})$ (Table 2) [21]. In addition, Yang et al. have carried out a more comprehensive sequencing analysis of TEX11 exons and flanking introns in a large number of patients with nonobstructive azoospermia and fertile controls. They identified a total of 40 different sequence variants of TEX11 in all subjects, but 22 different TEX11 mutations (including three singletons) were observed in fertile controls, suggesting that these mutations are not linked to spermatogenic failure [4]. Eighteen of the 21 singletons were found in patients with azoospermia, which included five exonic missense mutations (W117R, V142I, Q172R, T244I, and V748A), two exonic silent mutations $(405 \mathrm{C} \rightarrow \mathrm{T}$ and $2319 \mathrm{~T} \rightarrow \mathrm{C}$ ), one exonic frameshift mutation, and 10 intronic mutations (Table 2). Some subjects exhibit multiple TEX11 mutations. Interestingly, many intronic alterations such as $-48 \mathrm{G} \rightarrow \mathrm{A}$ and + $42 \mathrm{C} \rightarrow \mathrm{A}$ and silent mutations like $405 \mathrm{C} \rightarrow \mathrm{T}$ and $2319 \mathrm{~T} \rightarrow \mathrm{C}$ cause meiotic arrest, while some exonic missense mutations (K115R, M152 V, E436K, and 
Table 2 Mutations of TEX11 detected in patients with azoospermia*

\begin{tabular}{|c|c|c|c|c|c|}
\hline Position & Nucleotide change & Protein/RNA change & Spermatogenic failure & No. of patients & Ref. \\
\hline Exon 6 & $405 C \rightarrow T$ & Silent mutation, A135spl d ${ }^{\#}$ & Few sperm & 1 & 21 \\
\hline Exon 7 & $466 \mathrm{~A} \rightarrow \mathrm{G}$ & Missense mutation, M156V & No sperm & 1 & \\
\hline Exons 9-11 & 607del237bp & 203del79aa & Few sperm & 2 & \\
\hline Intron 10 & $748+1 \mathrm{G} \rightarrow \mathrm{A}^{+}$ & L249spl d \# & No sperm & 1 & \\
\hline Intron 21 & $1793+1 \mathrm{G} \rightarrow \mathrm{C}^{\dagger}$ & R597spl d \# & No sperm & 1 & \\
\hline Exon 24 & $2047 G \rightarrow A$ & Missense mutation, A683T & Few sperm & 1 & \\
\hline Exon 6 & 349T $\rightarrow \mathrm{A}$ & Missense mutation, W117R & No sperm & 1 & 5 \\
\hline Exon 6 & $405 C \rightarrow T$ & Silent mutation & No sperm & 1 & \\
\hline Exon 7 & $424 \mathrm{G} \rightarrow \mathrm{A}$ & Missense mutation, V142I & No sperm & 1 & \\
\hline Exon 7 & $515 \mathrm{~A} \rightarrow \mathrm{G}$ & Missense mutation, Q172R & No sperm & 1 & \\
\hline Exon 10 & $731 C \rightarrow T$ & Missense mutation, T244I & No sperm & 1 & \\
\hline Exon 16 & $1258 \operatorname{lns}(\mathrm{TT})$ & Frameshift mutation; 1258GATG $\rightarrow$ TTGGTA & No sperm & 1 & \\
\hline Exon 26 & $2243 T \rightarrow C$ & Missense mutation, V748A & No sperm & 1 & \\
\hline Exon 27 & $2319 \mathrm{~T} \rightarrow \mathrm{C}$ & Silent mutation & No sperm & 1 & \\
\hline Intron 3 & $-17 \mathrm{~T} \rightarrow \mathrm{C}^{+}$ & Intronic alteration & No sperm & 1 & \\
\hline Intron 5 & $-48 \mathrm{G} \rightarrow \mathrm{A}^{\dagger}$ & Intronic alteration & No sperm & 1 & \\
\hline Intron 10 & $+42 \mathrm{C} \rightarrow \mathrm{A}^{+}$ & Intronic alteration & No sperm & 1 & \\
\hline Intron 12 & $-28 \mathrm{~T} \rightarrow \mathrm{C}^{\dagger}$ & Intronic alteration & No sperm & 1 & \\
\hline Intron 15 & $-64 \mathrm{G} \rightarrow \mathrm{A}^{+}$ & Intronic alteration & No sperm & 1 & \\
\hline Intron 21 & $-1 \mathrm{G} \rightarrow \mathrm{A}^{\dagger}$ & Alteration of splicing acceptor site & No sperm & 1 & \\
\hline Intron 22 & $-37 \mathrm{~A} \rightarrow \mathrm{G}^{+}$ & Intronic alteration & No sperm & 1 & \\
\hline Intron 24 & $+119 \mathrm{G} \rightarrow \mathrm{A}^{+}$ & Intronic alteration & No sperm & 1 & \\
\hline Intron 27 & $-55 \mathrm{~A} \rightarrow \mathrm{C}^{+}$ & Intronic alteration & No sperm & 1 & \\
\hline Intron 28 & $-44 \mathrm{~A} \rightarrow \mathrm{G}^{+}$ & Intronic alteration & No sperm & 1 & \\
\hline Exon 29 & $2568 \mathrm{G} \rightarrow \mathrm{T}$ & Missense mutation, W856C & No sperm & 2 & This study \\
\hline
\end{tabular}

TEX11 mutations are mapped to isoform 2 (GenBank accession number, NM_031276)

\# The term spl d represents the splicing donor site

${ }^{\dagger}+1$ refers to the first base of a given intron, while -1 denotes the last base

D832E) have been found in fertile men [4]. In the present study, we identified one novel exonic missense TEX11 mutation (W856C) in two brothers but not their mother. Based on the testicular histology of two brothers, we observed a thicker basement membrane of the seminiferous tubules and poorly developed spermatocytes. No post-meiotic round spermatids or mature spermatozoa were observed in the seminiferous tubules, suggesting that this mutation will cause meiotic arrest. Moreover, Yatsenko et al. have observed that TEX11 expression is absent in the majority of seminiferous tubules but can be seen in rare tubules with remaining late spermatocytes and round spermatids [21]. In contrast, we found positive TEX11 staining in all seminiferous tubules, with strong expression in spermatogonia and weak expression in spermatocytes. This difference is probably due to different TEX11 mutation sites or antibody specificity. Although the precise causes for most TEX11 mutations remain unclear, Yang et al. have verified that one frameshift mutation of TEX11 in an azoospermic man with meiotic arrest was inherited from his mother because his mother was heterozygous for this mutation and his brother was azoospermic [4]. Interestingly, we observed the same TEX11 mutation in two brothers from a family without a history of infertility, whereas their mother had the wild-type allele of TEX11. The abundance of TEX11 mutations increases the difficulty in identifying which mutations will cause male infertility.

In conclusion, we identified one novel TEX11 mutation in two azoospermia brothers and summarized the literature regarding TEX11 mutations and male infertility. TEX11 mutations are closely related to male infertility, especially azoospermia. Auxiliary analyses such as testicular histology and family infertility history will also help to figure out the relationship between TEX11 mutations and male infertility. 


\section{Abbreviations}

FISH: Fluorescent in situ hybridization; PCR: Polymerase chain reaction; WES: Whole-exome sequencing

\section{Funding}

This study was supported by the Science and Technology Planning Project (Grant No. 3502Z20154033), the Major/Important Disease Research Project (Grant No. 3502Z20159022), and the Young/Middle-aged Talent Cultivation Project (Grant No. 2015-ZQN-JC-44). The authors declare no competing interest(s)

\section{Authors' contributions}

YS performed the experiment and wrote the manuscript; LZ performed immunohistochemistry and analyzed the data; ZJ and LM collected the samples; LD, SL and XW performed sequencing and analysis; XY collected the samples and analyzed the data; PL designed and supervised the experiment, reviewed and revised the manuscript. All authors read and approved the final manuscript.

\section{Ethics approval and consent to participate}

This study was approved by the Ethics Committee of Xiamen Women's and Children's Health Hospital.

\section{Consent for publication}

Written consent was obtained from both patients, their mother, and families of both donor testes to publish their data.

\section{Competing interests}

The authors declare that they have no competing interests.

\section{Publisher's Note}

Springer Nature remains neutral with regard to jurisdictional claims in published maps and institutional affiliations.

Received: 12 April 2016 Accepted: 23 March 2018

Published online: 16 April 2018

\section{References}

1. Matzuk MM, Lamb DJ. The biology of infertility: research advances and clinical challenges. Nat Med. 2008;14(11):1197-213.

2. Miyamoto T, Minase G, Okabe K, Ueda H, Sengoku K. Male infertility and its genetic causes. J Obstet Gynaecol Res. 2015;41(10):1501-5.

3. Silber SJ. Evaluation and treatment of male infertility. Clin Obstet Gynecol. 2000;43(4):854-88.

4. Yang F, Silber S, Leu NA, Oates RD, Marszalek JD, Skaletsky H, Brown LG, Rozen S, Page DC, Wang PJ. TEX11 is mutated in infertile men with azoospermia and regulates genome-wide recombination rates in mouse. EMBO Mol Med. 2015;7(9):1198-210.

5. Mueller JL, Mahadevaiah SK, Park PJ, Warburton PE, Page DC, Turner JM. The mouse $X$ chromosome is enriched for multicopy testis genes showing postmeiotic expression. Nat Genet. 2008;40(6):794-9.

6. Stouffs K, Seneca S, Lissens W. Genetic causes of male infertility. Ann Endocrinol (Paris). 2014;75(2):109-11.

7. Suganthi R, Vijesh W, Vandana N, Fathima Ali Benazir J. Y choromosoma microdeletion screening in the workup of male infertility and its current status in India. Int J Fertil Steril. 2014;7(4):253-66.

8. Zheng K, Yang F, Wang PJ. Regulation of male fertility by X-linked genes. J Androl. 2010;31(1):79-85

9. Cheng Y, Buffone MG, Kouadio M, Goodheart M, Page DC, Gerton GL, Davidson I, Wang PJ. Abnormal sperm in mice lacking the Taf7l gene. Mol Cell Biol. 2007;27(7):2582-9.

10. Pan J, Eckardt S, Leu NA, Buffone MG, Zhou J, Gerton GL, McLaughlin KJ, Wang PJ. Inactivation of Nxf2 causes defects in male meiosis and age-dependent depletion of spermatogonia. Dev Biol. 2009:330(1):167-74.

11. Blatch $G L$, Lässle M. The tetratricopeptide repeat: a structural motif mediating protein-protein interactions. BioEssays. 1999:21(11):932-9.

12. Chelysheva L, Gendrot G, Vezon D, Doutriaux MP, Mercier R, Grelon M. Zip4/ Spo22 is required for class I CO formation but not for synapsis completion in Arabidopsis thaliana. PLoS Genet. 2007;3(5):e83.
13. Yang F, Gell K, van der Heijden GW, Eckardt S, Leu NA, Page DC, Benavente R, Her C, Höög C, McLaughlin KJ, Wang PJ. Meiotic failure in male mice lacking an X-linked factor. Genes Dev. 2008;22(5):682-91.

14. Adelman CA, Petrini JH. ZIP4H (TEX11) deficiency in the mouse impairs meiotic double strand break repair and the regulation of crossing over. PLoS Genet. 2008:4(3):e1000042.

15. Wang PJ, McCarrey JR, Yang F, Page DC. An abundance of X-linked genes expressed in spermatogonia. Nat Genet. 2001;27:422-6.

16. Miki K, Willis WD, Brown PR, Goulding EH, Fulcher KD, Eddy EM. Targeted disruption of the Akap4 gene causes defects in sperm flagellum and motility. Dev Biol. 2002;248(2):331-42.

17. Nuti F, Krausz C. Gene polymorphisms/mutations relevant to abnormal spermatogenesis. Reprod BioMed Online. 2008;16(4):504-13.

18. Mifsud A, Sim CK, Boettger-Tong H, Moreira S, Lamb DJ, Lipshultz LI, Yong EL. Trinucleotide (CAG) repeat polymorphisms in the androgen receptor gene: molecular markers of risk for male infertility. Fertil Steril. 2001;75(2):275-81.

19. Li P, Ding L, Sha YW, Song YQ, Lin J, Werner EF, She M. Non-chimerism and chimerism pseudo dicentric $Y$ chromosome: two case reports about azoospermia and cytogenetic/molecular genetic analysis in the Chinese population. J Assist Reprod Genet. 2013;30(4):539-46.

20. Stouffs K, Lissens W. X chromosomal mutations and spermatogenic failure. Biochim Biophys Acta. 2012;1822(12):1864-72.

21. Yatsenko AN, Georgiadis AP, Röpke A, Berman AJ, Jaffe T, Olszewska M, Westernströer B, Sanfilippo J, Kurpisz M, Rajkovic A, et al. X-linked TEX11 mutations, meiotic arrest, and azoospermia in infertile men. N Engl J Med. 2015:372(22):2097-107.

22. Yu YH, Siao FP, Hsu LC, Yen PH. TEX11 modulates germ cell proliferation by competing with estrogen receptor $\beta$ for the binding to HPIP. Mol Endocrinol. 2012;26(4):630-42.

23. Tang L, Zeng W, Clark RK, Dobrinski I. Characterization of the porcine testisexpressed gene 11 (Tex11). Spermatogenesis. 2011;1(2):147-51.

24. Zhang $X$, Ding $M$, Ding $X, L i ~ T$, Chen $H$. Six polymorphisms in genes involved in DNA double-strand break repair and chromosome synapsis: association with male infertility. Syst Biol Reprod Med. 2015; 61(4):187-93

25. de Camargo GM, Porto-Neto LR, Kelly MJ, Bunch RJ, McWilliam SM, Tonhati H, Lehnert SA, Fortes MR, Moore SS. Non-synonymous mutations mapped to chromosome $X$ associated with andrological and growth traits in beef cattle. BMC Genomics. 2015;16:384.

26. Lyons RE, Loan NT, Dierens L, Fortes MR, Kelly M, McWilliam SS, Li Y, Bunch RJ, Harrison BE, Barendse W, et al. Evidence for positive selection of taurine genes within a QTL region on chromosome X associated with testicular size in Australian Brahman cattle. BMC Genet. 2014:15:6.

27. World Health Organization. Laboratory manual for the examination of human semen and sperm-cervical mucus interaction. 4th ed. New York: Cambridge University Press; 1999.

28. Sadik DI, Seifeldin NS. Fluorescence in situ hybridisation analysis of sex chromosome in non-obstructive azoospermic men. Andrologia. 2014; 46(3):231-9.

29. Simons A, Shaffer LG, Hastings RJ. Cytogenetic Nomenclature: Changes in the ISCN 2013 Compared to the 2009 Edition. Cytogenet Genome Res. 2013;141(1):1-6.

30. Ramasamy R, Bakırcıoğlu ME, Cengiz C, Karaca E, Scovell J, Jhangiani SN, Akdemir ZC, Bainbridge M, Yu Y, Huff C, et al. Whole-exome sequencing identifies novel homozygous mutation in NPAS2 in family with nonobstructive azoospermia. Fertil Steril. 2015:104(2):286-91.

31. Kolesnikova LI, Kolesnikov SI, Kurashova NA, Bairova TA. Causes and Factors of Male Infertility. Vestn Ross Akad Med Nauk. 2015;(5):579-84.

32. Reijo R, Lee TY, Salo P, Alagappan R, Brown LG, Rosenberg M, Rozen S, Jaffe T, Straus D, Hovatta O, et al. Diverse spermatogenic defects in humans caused by Y chromosome deletions encompassing a novel RNA-binding protein gene. Nat Genet. 1995;10(4):383-93.

33. Tüttelmann F, Gromoll J. Novel genetic aspects of Klinefelter's syndrome. Mol Hum Reprod. 2010;16(6):386-95. 